\title{
A QUADRATIC REGRESSION PROBLEM FOR TWO-STATE ALGEBRAS WITH APPLICATION TO THE CENTRAL LIMIT THEOREM
}

\author{
MAREK BOŻEJKO AND WŁODZIMIERZ BRYC
}

\begin{abstract}
We extend a free version [1, Theorem 3.2] of the LahaLukacs theorem to probability spaces with two-states. We then use this result to generalize a noncommutative CLT of Kargin [7] to the two-state setting.
\end{abstract}

\section{INTRODUCTION}

1.1. A two-state freeness condition. Let $\mathcal{A}$ be a unital *-algebra with two states $\psi, \varphi: \mathcal{A} \rightarrow \mathbb{C}$. We assume that both states fulfill the usual assumptions of positivity and normalization, and we assume tracial property $\psi(a b)=\psi(b a)$ for $\psi$, but not for $\varphi$.

A self-adjoint element $\mathbb{X} \in \mathcal{A}$ with moments that fulfill appropriate growth condition defines a pair $\mu, \nu$ of probability measures on $(\mathbb{R}, \mathcal{B})$ such that

$$
\varphi\left(\mathbb{X}^{k}\right)=\int_{\mathbb{R}} x^{k} \mu(d x) \text { and } \psi\left(\mathbb{X}^{k}\right)=\int_{\mathbb{R}} x^{k} \nu(d x) .
$$

We will refer to measures $\mu, \nu$ as the $\varphi$-law and the $\psi$-law of $\mathbb{X}$, respectively.

With each set of $a_{1}, \ldots, a_{n} \in \mathcal{A}$ and a pair of states we associate the cumulants $R_{k}=R_{k, \varphi, \psi}, k=1,2, \ldots$, which are the multilinear functions $\mathcal{A}^{k} \rightarrow \mathbb{C}$ defined by

$$
\begin{aligned}
& \text { (1.1) } \varphi\left(a_{1} a_{2} \ldots a_{n}\right) \\
& =\sum_{k=1}^{n} \sum_{1=s_{1}<s_{2}<\cdots<s_{k} \leq n} R_{k}\left(a_{1}, a_{s_{2}}, \ldots, a_{s_{k}}\right) \varphi\left(a_{s_{k}+1} \ldots a_{n}\right) \prod_{r=1}^{k-1} \psi\left(\prod_{j=s_{r}+1}^{s_{r+1}-1} a_{j}\right) .
\end{aligned}
$$

Date: Printed: February 18, 2019. File: noncom-clt-07-5.TEX.

2000 Mathematics Subject Classification. Primary: 46L53; Secondary: 60E05, $05 \mathrm{~A} 18$.

Key words and phrases. generalized two-state freeness, generalized free Meixner distribution, Laha-Lukacs theorem, noncommutative quadratic regression.

Research partially supported by the Taft Research Center, KBN Grant No 1 PO3A 01330, and NSF grant \#DMS-0504198. 
We will use the notation

$$
r_{n}\left(a_{1}, \ldots, a_{n}\right):=R_{n, \psi, \psi}\left(a_{1}, \ldots, a_{n}\right) .
$$

We remark that $r_{n}$ are the free cumulants with respect to state $\psi$, as defined by Speicher [12, 13]; see also [11]. For more general theory of cumulants see [10].

Fix $a \in \mathcal{A}$, and consider the following formal power series

$$
\begin{aligned}
R(z) & =\sum_{n=1}^{\infty} R_{n}(a, \ldots, a) z^{n-1} \\
m(z) & =\sum_{n=0}^{\infty} z^{n} \psi\left(a^{n}\right), \\
M(z) & =\sum_{n=0}^{\infty} z^{n} \varphi\left(a^{n}\right) .
\end{aligned}
$$

By [2, Theorem 5.1], Eqtn. (1.1) is equivalent to the following relation

$$
M(z)(1-z R(z m(z)))=1 \text {. }
$$

Definition 1.1. We say that subalgebras $\mathcal{A}_{1}, \mathcal{A}_{2}, \ldots$ are $(\varphi \mid \psi)$-free if for every choice of $a_{1}, \ldots, a_{n} \in \bigcup_{j} \mathcal{A}_{j}$ we have

$$
R_{n}\left(a_{1}, \ldots, a_{n}\right)=0 \text { except if all } a_{j} \text { come from the same algebra. }
$$

When the algebras are $(\psi \mid \psi)$-free, we will abbreviate this to $\psi$-free. From [12] it follows that $\psi$-freeness coincides with the usual concept of freeness as introduced by Voiculescu [14.

We will say that $\mathbb{X}, \mathbb{Y}$ are $(\varphi \mid \psi)$-free if the unital algebras $\mathbb{C}\langle\mathbb{X}\rangle$ and $\mathbb{C}\langle\mathbb{Y}\rangle$ are $(\varphi \mid \psi)$-free.

A related concept is the following.

Definition 1.2 ([3] and [2]). We say that subalgebras $\mathcal{A}_{1}, \mathcal{A}_{2}, \ldots$ are $c$-free if for every choice of $i_{1} \neq i_{2} \neq \cdots \neq i_{n}$ and every choice of $a_{j} \in \mathcal{A}_{j}$ such that $\psi\left(a_{j}\right)=0$ (thus $a_{j} \neq 1$ ) we have

$$
\varphi\left(a_{i_{1}} \ldots a_{i_{n}}\right)=\prod_{k=1}^{n} \varphi\left(a_{i_{k}}\right) .
$$

1.2. Properties of $(\varphi \mid \psi)$-freeness. If $\mathcal{A}_{1}, \mathcal{A}_{2}$ are $(\varphi \mid \psi)$-free then for $a \in \mathcal{A}_{1}, b \in \mathcal{A}_{2}$

$$
\varphi(a b)=\varphi(a) \varphi(b)
$$

For $a_{1}, a_{2} \in \mathcal{A}_{1}, b \in \mathcal{A}_{2}$ we have

$$
\varphi\left(a_{1} b a_{2}\right)=\psi(b) \varphi\left(a_{1} a_{2}\right)-\psi(b) \varphi\left(a_{1}\right) \varphi\left(a_{2}\right)+\varphi(b) \varphi\left(a_{1}\right) \varphi\left(a_{2}\right) .
$$


For $a_{1}, a_{2} \in \mathcal{A}_{1}, b_{1}, b_{2} \in \mathcal{A}_{2}$ we have

$$
\begin{aligned}
\varphi\left(a_{1} b_{1} a_{2} b_{2}\right) & =\varphi\left(a_{1} a_{2}\right) \psi\left(b_{1}\right) \psi\left(b_{2}\right)-\varphi\left(a_{1}\right) \psi\left(a_{2}\right) \varphi\left(b_{1} b_{2}\right) \\
& +\varphi\left(a_{1}\right) \psi\left(a_{2}\right) \varphi\left(b_{1}\right) \varphi\left(b_{2}\right)-\varphi\left(a_{1}\right) \varphi\left(a_{2}\right) \varphi\left(b_{1}\right) \varphi\left(b_{2}\right)
\end{aligned}
$$

Formulas (1.8) (1.9) and are identical to formulas under $c$-freeness as given in [2, Lemma 2.1]. Together with formula (1.10) they imply that for a pair of $(\varphi \mid \psi)$-free algebras, (1.7) holds for $n \leq 4$. One can check that if $a, b$ are $(\varphi \mid \psi)$-free and $\psi(a)=\psi(b)=0$ but $\psi(b a b) \neq 0$ then $\varphi(a b a b a) \neq \varphi(a)^{3} \varphi(b)^{2}$; thus the concepts of $c$-freeness and of $(\varphi \mid \psi)$-freeness are not equivalent. Nevertheless they coincide for $\psi$-free algebras as noted in the following.

Lemma 1.1 ([2], page 368). Suppose $\mathcal{A}_{1}, \mathcal{A}_{2}, \ldots$ are $\psi$-free. Then the algebras $\mathcal{A}_{1}, \mathcal{A}_{2}, \ldots$ are $(\varphi \mid \psi)$-free if and only if they are $c$-free.

We will also rely on the following fact.

Lemma $1.2([2])$. Given a noncommutative random variable $\mathbb{X}$ in a two-state probability space, there exist a two-state algebra (which one can take as the algebra of noncommutative polynomials $\mathbb{C}\langle\mathbb{X}, \mathbb{Y}\rangle$ in two variables) and two non-commutative random variables $\widetilde{\mathbb{X}}, \widetilde{\mathbb{Y}}$ which are $\psi$-free, $(\varphi \mid \psi)$-free, and both have the same $\varphi$-law and $\psi$-law as $\mathbb{X}$.

Proof. Ref. [3, Theorem 1], see also [2, Theorem 2.2], shows how to extend both states to the free product of the original algebra so that the resulting algebras are $c$-free and $\psi$-free. By Lemma 1.1, they are thus $(\varphi \mid \psi)$-free.

\section{A $(\varphi \mid \psi)$-FREE QUADRATIC REGRESSION PROBLEM}

In this section we prove a two-state version of [1, Theorem 3.2]. The statement is fairly technical, but we found it useful for our proof of the Central Limit Theorem (Theorem 4.1 below).

Theorem 2.1. Suppose $\mathbb{X}, \mathbb{Y}$ are self-adjoint $(\varphi \mid \psi)$-free and

$$
\varphi\left(\mathbb{X}^{n}\right)=\varphi\left(\mathbb{Y}^{n}\right), \psi\left(\mathbb{X}^{n}\right)=\psi\left(\mathbb{Y}^{n}\right)
$$

for all $n$. Furthermore, assume that $\varphi(\mathbb{X})=0, \varphi\left(\mathbb{X}^{2}\right)=1$. (This can always be achieved by a shift and dilation, as long as $\varphi\left(\mathbb{X}^{2}\right) \neq 0$.)

Let $\mathbb{S}=\mathbb{X}+\mathbb{Y}$ and suppose that there are $a, c \in \mathbb{R}$ and $b>-2$ such that

$$
\varphi\left((\mathbb{X}-\mathbb{Y})^{2} \mathbb{S}^{n}\right)=c \varphi\left(\left(4 \mathbb{I}+2 a \mathbb{S}+b \mathbb{S}^{2}\right) \mathbb{S}^{n}\right), n=0,1,2 \ldots
$$


Then the $\varphi$-moment generating functions $M_{\mathbb{S}}(z):=\sum_{k=0}^{\infty} z^{k} \varphi\left(\mathbb{S}^{k}\right)$ and $m_{\mathbb{S}}(z):=\sum_{k=0}^{\infty} z^{k} \psi\left(\mathbb{S}^{k}\right)$, which are defined as formal power series, are related as follows

$$
M_{\mathbb{S}}(z)=\frac{2+b-(2 a z+b) m_{\mathbb{S}}(z)}{2+b-\left(4 z^{2}+2 a z+b\right) m_{\mathbb{S}}(z)} .
$$

Remark 2.1. We will apply (2.3) to the case when $m_{\mathbb{S}}(z)$ converges for small enough $|z|$, in the form as written. In general, the right hand side of (2.3) needs to be interpreted correctly. Recall that the composition $p(q(z))$ of two power series $p, q$ is well defined if $q(z)$ has no constant term. Note that the formal power series $-b+\left(4 z^{2}+2 a z+b\right) m_{\mathbb{S}}(z)$ has no constant term, so it can be composed with the formal power series $\sum_{n=0}^{\infty} \frac{1}{2^{n+1}} z^{n}$, which is a formal power expansion of the function $\frac{1}{2-z}$. It is therefore natural to denote such a composition by

$$
\frac{1}{2-\left(-b+\left(4 z^{2}+2 a z+b\right) m_{\mathbb{S}}(z)\right)} \text {. }
$$

The right hand side of (2.3) is then interpreted as the product of this power series with the formal power series $2+b-(2 a z+b) m_{\mathbb{S}}(z)$.

Remark 2.2. Our assumptions on $\varphi$ do not allow us to use conditional expectations. However, it is still natural to ask which properties of conditional expectations would have implied assumptions of Theorem 2.1. To this end, we denote by $\varphi(\cdot \mid \mathbb{S})$ the conditional expectation onto the commutative algebra generated by $\mathbb{S}$.

From equality of the laws (2.1) and $(\varphi \mid \psi)$-freeness, one can deduce that

$$
\varphi\left(\mathbb{X} \mathbb{S}^{n}\right)=\frac{1}{2} \varphi\left(\mathbb{S}^{n+1}\right), n=0,1,2 \ldots
$$

(See (2.7) below.) When the conditional expectation exists, this property follows from $\varphi(\mathbb{X} \mid \mathbb{S})=\frac{1}{2} \mathbb{S}$. We can then derive (2.2) from the quadratic variance property

$$
\varphi\left(\mathbb{X}^{2} \mid \mathbb{S}\right)-(\varphi(\mathbb{X} \mid \mathbb{S}))^{2}=c\left(\mathbb{I}+\frac{a}{2} \mathbb{S}+\frac{b}{4} \mathbb{S}^{2}\right)
$$

2.1. Proof of Theorem 2.1. We first remark that $c=(2+b)^{-1}$. This follows from (2.2) with $n=0$ since $\varphi(\mathbb{X} \pm \mathbb{Y})^{2}=2 \pm \varphi(\mathbb{X} \mathbb{Y}) \pm \varphi(\mathbb{Y} \mathbb{X})=2$.

By definition, $R_{n}(\mathbb{S}, \ldots, \mathbb{S})=R_{n}(\mathbb{X}, \ldots, \mathbb{X})+R_{n}(\mathbb{Y}, \ldots, \mathbb{Y})$. From (2.1) we see that $R_{n}(\mathbb{X}, \ldots, \mathbb{X})=R_{n}(\mathbb{Y}, \ldots, \mathbb{Y})$. Thus

$$
\begin{array}{r}
R_{n}(\mathbb{X}-\mathbb{Y}, \mathbb{S}, \ldots, \mathbb{S})=R_{n}(\mathbb{X}, \mathbb{S}, \ldots, \mathbb{S})-R_{n}(\mathbb{Y}, \mathbb{S}, \ldots, \mathbb{S}) \\
=R_{n}(\mathbb{X}, \ldots, \mathbb{X})-R_{n}(\mathbb{Y}, \ldots, \mathbb{Y})=0
\end{array}
$$


for all $n$. By (1.1) this implies

$$
\varphi\left((\mathbb{X}-\mathbb{Y}) \mathbb{S}^{n}\right)=0 .
$$

Similarly, using multilinearity of $R$,

$$
\begin{aligned}
& R_{n}(\mathbb{X}-\mathbb{Y}, \mathbb{X}-\mathbb{Y}, \mathbb{S}, \ldots, \mathbb{S}) \\
& =R_{n}(\mathbb{X}, \mathbb{X}-\mathbb{Y}, \mathbb{S}, \ldots, \mathbb{S})-R_{n}(\mathbb{Y}, \mathbb{X}-\mathbb{Y}, \mathbb{S}, \ldots, \mathbb{S}) \\
& \quad=R_{n}(\mathbb{X}, \ldots, \mathbb{X})+R_{n}(\mathbb{Y}, \ldots, \mathbb{Y})=R_{n}(\mathbb{S}, \ldots, \mathbb{S})
\end{aligned}
$$

for all $n \geq 2$. Formula (1.1) therefore implies that

$$
\text { (2.9) } \varphi\left((\mathbb{X}-\mathbb{Y})^{2} \mathbb{S}^{n}\right)
$$

$$
\begin{aligned}
& =\sum_{k=2}^{n+2} \sum_{1=b_{1}<b_{2}=2<\cdots<b_{k} \leq n+2} R_{k}(\mathbb{X}-\mathbb{Y}, \mathbb{X}-\mathbb{Y}, \mathbb{S} \ldots, \mathbb{S}) \varphi\left(\mathbb{S}^{n-b_{k}-1}\right) \prod_{r=1}^{k-1} \psi\left(\mathbb{S}^{b_{r+1}-b_{r}-1}\right) \\
& +\sum_{k=1}^{n+2} \sum_{1=b_{1}<2<b_{2}<\cdots<b_{k} \leq n+2} R_{k}(\mathbb{X}-\mathbb{Y}, \mathbb{S}, \ldots, \mathbb{S}) \varphi\left(\mathbb{S}^{n-b_{k}-1}\right) \prod_{r=1}^{k-1} \psi\left(\mathbb{S}^{b_{r+1}-b_{r}-1}\right) .
\end{aligned}
$$

By (2.6), the second sum vanishes. Using (2.8) we get

$$
\begin{aligned}
& (2.10) \varphi\left((\mathbb{X}-\mathbb{Y})^{2} \mathbb{S}^{n}\right) \\
& =\sum_{k=2}^{n+2} \sum_{1=b_{1}<b_{2}=2<\cdots<b_{k} \leq n+2} R_{k}(\mathbb{S}, \mathbb{S}, \mathbb{S} \ldots, \mathbb{S}) \varphi\left(\mathbb{S}^{n-b_{k}-1}\right) \prod_{r=1}^{k-1} \psi\left(\mathbb{S}^{b_{r+1}-b_{r}-1}\right) .
\end{aligned}
$$

Comparing this with the decomposition for $\varphi\left(\mathbb{S}^{n+2}\right)$ we see that

$$
-\sum_{k=2}^{n+2} \sum_{1=b_{1}<2<b_{2}<\cdots<b_{k} \leq n+2} R_{k}(\mathbb{S}, \mathbb{S}, \ldots, \mathbb{S}) \varphi\left(\mathbb{S}^{n-b_{k}-1}\right) \prod_{r=1}^{k-1} \psi\left(\mathbb{S}^{b_{r+1}-b_{r}-1}\right) .
$$

We now rewrite the last sum based on the value of $m=b_{2}-b_{1}$, compare [1]. We have

$$
-\sum_{m=1}^{n} \psi\left(\mathbb{S}^{m}\right) \sum_{k=2}^{n+2} \sum_{1=b_{1}<1+m=b_{2}<\cdots<b_{k} \leq n+2} R_{k}(\mathbb{S}, \mathbb{S}, \ldots, \mathbb{S}) \varphi\left(\mathbb{S}^{n-b_{k}-1}\right) \prod_{r=1}^{k-1} \psi\left(\mathbb{S}^{b_{r+1}-b_{r}-1}\right) .
$$


Since $b_{2}-b_{1}-1=m$, formula (2.8) gives

$$
\begin{aligned}
& \sum_{k=2}^{n+2} \sum_{1=b_{1}<1+m=b_{2}<\cdots<b_{k} \leq n+2} R_{k}(\mathbb{S}, \mathbb{S}, \ldots, \mathbb{S}) \varphi\left(\mathbb{S}^{n-b_{k}-1}\right) \prod_{r=1}^{k-1} \psi\left(\mathbb{S}^{b_{r+1}-b_{r}-1}\right) \\
= & \sum_{k=2}^{n+2} R_{k}(\mathbb{X}-\mathbb{Y}, \mathbb{X}-\mathbb{Y}, \mathbb{S}, \ldots, \mathbb{S}) \varphi\left(\mathbb{S}^{n-b_{k}-1}\right) \psi\left(\mathbb{S}^{m}\right) \\
\times \prod_{r=2} \psi\left(\prod_{1}<1+m=b_{2}<\cdots<b_{k} \leq n+2\right. & \left.\mathbb{S}^{b_{r+1}-b_{r}-1}\right) .
\end{aligned}
$$

Re-indexing the variables so that $b_{2}=2$ and inserting this into (2.10) we get

$$
\varphi\left((\mathbb{X}-\mathbb{Y})^{2} \mathbb{S}^{n}\right)=\varphi\left(\mathbb{S}^{n+2}\right)-\sum_{m=1}^{n} \psi\left(\mathbb{S}^{m}\right) \varphi\left((\mathbb{X}-\mathbb{Y})^{2} \mathbb{S}^{n-m}\right) .
$$

Thus from (2.2) we get

$$
\varphi\left(\mathbb{S}^{n+2}\right)=\frac{1}{2+b} \sum_{j=0}^{n} \psi\left(\mathbb{S}^{j}\right)\left(4 \varphi\left(\mathbb{S}^{n-j}\right)+2 a \varphi\left(\mathbb{S}^{n-j+1}\right)+b \varphi\left(\mathbb{S}^{n-j+2}\right)\right) .
$$

A routine argument now relates the formal power series:

$$
M_{\mathbb{S}}(z)=1+z^{2} \sum_{n=0}^{\infty} z^{n} \varphi\left(\mathbb{S}^{n+2}\right)
$$

$$
\begin{gathered}
=1+\frac{z^{2}}{2+b} \sum_{n=0}^{\infty} \sum_{j=0}^{n} z^{j} \psi\left(\mathbb{S}^{j}\right) z^{n-j}\left(4 \varphi\left(\mathbb{S}^{n-j}\right)+2 a \varphi\left(\mathbb{S}^{n-j+1}\right)+b \varphi\left(\mathbb{S}^{n-j+2}\right)\right) \\
=1+\frac{z^{2}}{2+b} \sum_{j=0}^{\infty} z^{j} \psi\left(\mathbb{S}^{j}\right) \sum_{n=j}^{\infty} z^{n-j}\left(4 \varphi\left(\mathbb{S}^{n-j}\right)+2 a \varphi\left(\mathbb{S}^{n-j+1}\right)+b \varphi\left(\mathbb{S}^{n-j+2}\right)\right) \\
=1+\frac{m_{\mathbb{S}}(z)}{2+b}\left(4 z^{2} M_{\mathbb{S}}(z)+2 a z\left(M_{\mathbb{S}}(z)-1\right)+b\left(M_{\mathbb{S}}(z)-1\right)\right) .
\end{gathered}
$$

\section{THE $\varphi$-LAW OF $\mathbb{X}$}

In this section we are interested in one explicit case when Theorem 2.1 allows us to determine the $\varphi$-law of $\mathbb{X}$ from the $\psi$-law of $\mathbb{X}$. This case arises when $\mathbb{X}, \mathbb{Y}$ are $\psi$-free and $(\varphi \mid \psi)$-free with compactly supported laws. Then the $\varphi$-law and the $\psi$-law of $\mathbb{X}+\mathbb{Y}$ are determined uniquely from the laws of $\mathbb{X}, \mathbb{Y}$ by the generalized convolution 
$\circledast$ which was introduced by Bożejko and Speicher [3] and studied in Refs. [2, 4, 5, 8, 9]. The generalized convolution is a binary operation on the pairs of compactly supported probability measures $(\mu, \nu)$. The analytic approach from Theorem 5.2 in Ref. [2] is especially convenient for explicit calculations. According to this result, the generalized convolution $\left(\mu_{1}, \nu_{1}\right) \circledast\left(\mu_{2}, \nu_{2}\right)$ of pairs of compactly supported probability measures is a pair $(\mu, \nu)$ of compactly supported probability measures which is determined by the following procedure. Consider the Cauchy transforms

$$
G_{j}(z)=\int \frac{1}{z-x} \mu_{j}(d x), g_{j}(z)=\int \frac{1}{z-x} \nu_{j}(d x), j=1,2 .
$$

Let $k_{j}(z)$ be the inverse function of $g_{j}(z)$ in a neighborhood of $\infty$, and define

$$
r_{j}(z)=k_{j}(z)-1 / z .
$$

On the second component the $c$-convolution acts as the free convolution, $\nu=\nu_{1} \boxplus \nu_{2}$. Recall [14] that the free convolution $\nu$ of measures $\nu_{1}, \nu_{2}$ is the unique probability measure with the Cauchy transform $g(z)$ which solves the equation

$$
g(z)=\frac{1}{z-r_{1}(g(z))-r_{2}(g(z))} .
$$

To define the action of the generalized convolution on the first component, let

Thus

$$
R_{j}(z)=k_{j}(z)-1 / G_{j}\left(k_{j}(z)\right)
$$

The first component of the generalized convolution is defined as the unique probability measure $\mu$ with the Cauchy transform

$$
G(z)=\frac{1}{z-R_{1}(g(z))-R_{2}(g(z))} .
$$

We write

$$
(\mu, \nu)=\left(\mu_{1}, \nu_{1}\right) \circledast\left(\mu_{2}, \nu_{2}\right) .
$$

We remark that

$$
r(z)=\sum_{k=1}^{\infty} r_{k} z^{k-1}, R(z)=\sum_{k=1}^{\infty} R_{k} z^{k-1}
$$

are generating functions for the $\psi$-free and $(\varphi \mid \psi)$-free cumulants respectively, see (1.3). We also note that the above relations can be interpreted as combinatorial relations between $\psi$-moments and $\varphi$-moments; 
the assumption of compact support allows us to determine the laws uniquely from moments.

\subsection{The case of "constant conditional variance".}

Proposition 3.1. Suppose $\mathbb{X}, \mathbb{Y}$ are $\psi$-free with the same compactly supported $\psi$-law $\nu$, and are $(\varphi \mid \psi)$-free with the same $\varphi$-law. If (2.2) holds with $a=b=0$, then the $\varphi$-law of $\mathbb{X}$ is compactly supported and uniquely determined by $\nu$.

Proof. The $\psi$-law of $\mathbb{S}$ is the free convolution $\nu \boxplus \nu$, so it is compactly supported. Therefore $m_{\mathbb{S}}(z)$ is given by a series that converges for small enough $|z|$. Then (2.3) reduces to

$$
M_{\mathbb{S}}(z)=\frac{1}{1-2 z^{2} m_{\mathbb{S}}(z)},
$$

and $M_{\mathbb{S}}(z)$ is also given by a convergent series. In particular, the $\varphi$-law of $\mathbb{S}$ is compactly supported. So for $\Im z>0$, the Cauchy transform is

$$
G_{\mathbb{S}}(z)=\frac{1}{z} M_{\mathbb{S}}(1 / z)=\frac{1}{z-2 g_{\mathbb{S}}(z)} .
$$

Thus $R_{k}(\mathbb{S}, \ldots, \mathbb{S})=0$ for all $k$ except for $R_{2}(\mathbb{S}, \mathbb{S})=2$. This shows that $R_{k}(\mathbb{X}, \ldots, \mathbb{X})=0$ for all $k$ except for $R_{2}(\mathbb{X}, \mathbb{X})=1$. Thus $R_{\mathbb{X}}(z)=z$ and (1.6) gives

$$
M_{\mathbb{X}}(z)=\frac{1}{1-z^{2} m_{\mathbb{X}}(z)} .
$$

This implies that $\varphi$-law of $\mathbb{X}$ has compact support, and its Cauchy transform is uniquely determined by

$$
G_{\mathbb{X}}(z)=\frac{1}{z-g_{\mathbb{X}}(z)} .
$$

In particular, suppose $\nu$ is the semicircle law with mean zero and variance $\sigma^{2}$, so that $g_{\mathbb{X}}(z)=\frac{z-\sqrt{z^{2}-4 \sigma^{2}}}{2 \sigma^{2}}$. Proposition 3.1 then shows that the $\varphi$-law of $\mathbb{X}$ has Cauchy-Stieltjes transform

$$
G_{\mathbb{X}}(z)=\frac{\left(\sigma^{2}-\frac{1}{2}\right) z-\frac{1}{2} \sqrt{z^{2}-4 \sigma^{2}}}{1+\left(\sigma^{2}-1\right) z^{2}} .
$$

This law plays the role of the "Gaussian limit" in [2]. 
3.2. The case of "linear conditional variance". Suppose (2.2) holds with $b=0$. Then (2.3) reduces to

$$
M_{\mathbb{S}}(z)=\frac{1-a z m_{\mathbb{S}}(z)}{1-(2 z+a) z m_{\mathbb{S}}(z)} .
$$

So again the $\Phi$-law of $\mathbb{S}$ is compactly supported, if the $\psi$-law is, and the Cauchy transform is

$$
G_{\mathbb{S}}(z)=\frac{1-a g_{\mathbb{S}}(z)}{z-(2+a z) g_{\mathbb{S}}(z)}=\frac{1}{z-R_{\mathbb{S}}\left(g_{\mathbb{S}}(z)\right)}
$$

with

$$
R_{\mathbb{S}}(u)=\frac{2 u}{1-a u}
$$

This shows that $R_{\mathbb{X}}(z)=\frac{z}{1-a z}$ and

$$
G_{\mathbb{X}}(z)=\frac{1-a g_{\mathbb{X}}(z)}{z-(1+a z) g_{\mathbb{X}}(z)} .
$$

In particular, suppose that the $\psi$-law of $\mathbb{X}$ is Marchenko-Pastur with parameter $\lambda>0$, so that

$$
g_{\mathbb{X}}(z)=\frac{z+(1-\lambda)-\sqrt{(z-1-\lambda)^{2}-4 \lambda}}{2 z} .
$$

If $a=1$, then the $\varphi$-law of $\mathbb{X}$ is compactly supported, with Cauchy transform

$$
G_{\mathbb{X}}(z)=\frac{1+\lambda+z(-1+2 \lambda)-\sqrt{(1-z+\lambda)^{2}-4 \lambda}}{2\left(1+z^{2}(-1+\lambda)+z(1+\lambda)\right)} .
$$

Related laws appear in [6, Eqtn. (17)] and in [2, page 380].

\section{Central Limit Theorem for non-identical summands}

The Central Limit Theorem and the Poisson convergence theorem for sums of $(\varphi \mid \psi)$-free random variables that are also $\psi$-free appear in [2, Theorems 4.3 and 4.4]. Recently Kargin [7] observed that in the free case one can dispense with the assumption of identical laws and at the same time relax the freeness assumption. In this section we use Theorem 2.1 to deduce a $(\varphi \mid \psi)$-free version of Kargin's result.

Definition 4.1. We will say that a sequence of random variables $\mathbb{X}_{1}, \mathbb{X}_{2}, \ldots$ satisfies Kargin's Condition A with respect to $(\varphi \mid \psi)$ if: 
(i) For every $k \notin\left\{j_{1}, \ldots, j_{n}\right\}$ the following singleton conditions hold:

$$
\begin{gathered}
\varphi\left(\mathbb{X}_{k} \mathbb{X}_{j_{1}} \ldots \mathbb{X}_{j_{r}}\right)=\varphi\left(\mathbb{X}_{j_{1}} \mathbb{X}_{k} \mathbb{X}_{j_{2}} \ldots \mathbb{X}_{j_{r}}\right)=\ldots \\
=\varphi\left(\mathbb{X}_{j_{1}} \ldots \mathbb{X}_{j_{r}} \mathbb{X}_{k}\right)=0 \\
\psi\left(\mathbb{X}_{k} \mathbb{X}_{j_{1}} \ldots \mathbb{X}_{j_{r}}\right)=0
\end{gathered}
$$

(In particular, $\psi\left(\mathbb{X}_{j}\right)=\varphi\left(\mathbb{X}_{j}\right)=0$.)

(ii) For every $k \notin\left\{j_{1}, \ldots, j_{r}\right\}$, and $0 \leq p \leq r$,

$$
\begin{aligned}
\varphi\left(\mathbb{X}_{k} \mathbb{X}_{j_{1}} \ldots \mathbb{X}_{j_{p}} \mathbb{X}_{k} \mathbb{X}_{j_{p+1}} \ldots \mathbb{X}_{j_{r}}\right) \\
=\varphi\left(\mathbb{X}_{k}^{2}\right) \psi\left(\mathbb{X}_{j_{1}} \ldots \mathbb{X}_{j_{p}}\right) \varphi\left(\mathbb{X}_{j_{p+1}} \ldots \mathbb{X}_{j_{r}}\right) .
\end{aligned}
$$

We remark that conditions (4.1) and (4.3) are automatically satisfied if $\mathbb{X}_{1}, \mathbb{X}_{2}, \ldots$ are $\varphi$-centered and $(\varphi \mid \psi)$-free; clearly, condition (4.2) holds true if $\mathbb{X}_{1}, \mathbb{X}_{2}, \ldots$ are $\psi$-centered and $\psi$-free but of course it is weaker and can hold also for classical (commutative) independent random variables.

Theorem 4.1. Suppose that

(i) $\mathbb{X}_{1}, \mathbb{X}_{2}, \ldots$ satisfy Kargin's Condition $A$ with respect to $(\varphi \mid \psi)$;

(ii) All joint moments of order $k$ are uniformly bounded

$$
\sup _{j_{1}, \ldots, j_{k} \geq 1}\left|\varphi\left(\mathbb{X}_{j_{1}} \ldots \mathbb{X}_{j_{k}}\right)\right| \leq C_{k}<\infty \text { for } k=1,2, \ldots
$$

(iii) Sequences $s_{j}^{2}:=\psi\left(\mathbb{X}_{j}^{2}\right)$ and $S_{j}^{2}:=\varphi\left(\mathbb{X}_{j}^{2}\right)$ satisfy

$$
\left(s_{1}^{2}+\cdots+s_{n}^{2}\right) / n \rightarrow s \text { and }\left(S_{1}^{2}+\cdots+S_{n}^{2}\right) / n \rightarrow S .
$$

(iv) $0<s, S<\infty$.

(v) The $\psi$-moments of $\frac{1}{\sqrt{s_{1}^{2}+\cdots+s_{n}^{2}}} \sum_{j=1}^{n} \mathbb{X}_{j}$ converge to the corresponding moments of a compactly supported probability measure $\nu$.

Then the $\varphi$-moments of $\frac{1}{\sqrt{S_{1}^{2}+\cdots+S_{n}^{2}}} \sum_{j=1}^{n} \mathbb{X}_{j}$ converges to the moments of the unique compactly supported law $\mu$ with Cauchy transform (3.6), where $g_{\mathbb{X}}(z)=\int \frac{S}{S z-s x} \nu(d x)$.

Combining Theorem 4.1 with [7] and formula (3.7) we get the following generalization of [2, Theorem 4.3].

Corollary 4.2. Suppose that

(i) $\mathbb{X}_{1}, \mathbb{X}_{2}, \ldots$ satisfy Kargin's Condition $A$ with respect to $(\varphi \mid \psi)$ and with respect to $(\psi \mid \psi)$. 
(ii) All moments are uniformly bounded: (4.4) holds true, and $\sup _{n}\left|\psi\left(\mathbb{X}_{n}^{k}\right)\right|<\infty$ for $k=1,2, \ldots$

(iii) $s_{j}^{2}:=\psi\left(\mathbb{X}_{j}^{2}\right)=s_{j}^{2}$ and $S_{j}^{2}:=\varphi\left(\mathbb{X}_{j}^{2}\right)$ satisfy (4.5) with $0<s, S<$ $\infty$.

Then the $\varphi$-law of $\frac{1}{\sqrt{S_{1}^{2}+\cdots+S_{n}^{2}}} \sum_{j=1}^{n} \mathbb{X}_{j}$ converges to the law $\mu$ with the Cauchy-Stieltjes transform (3.7) and $\sigma=s / S$.

4.1. Proof of Theorem 4.1. By [3] without loss of generality we may assume that we have a two-state probability space with two copies of the original sequence: $\left(\mathbb{X}_{k}\right)$ and $\left(\mathbb{Y}_{k}\right)$ each of them separately having the same $\psi$-moments and $\varphi$-moments as the original sequence, but such that the algebras $\mathcal{A}_{\mathbb{X}}$ and $\mathcal{A}_{\mathbb{Y}}$ generated by $\left(\mathbb{X}_{k}\right)$ and by $\left(\mathbb{Y}_{k}\right)$, respectively, are $\psi$-free and $(\varphi \mid \psi)$-free.

Under this representation, the $\psi$-distribution of $\frac{1}{\sqrt{s_{1}^{2}+\cdots+s_{n}^{2}}} \sum_{j=1}^{n}\left(\mathbb{X}_{j}+\right.$ $\mathbb{Y}_{j}$ ) converges to $\nu \boxplus \nu$. Our goal is to show that the $\varphi$-distribution of $\frac{1}{\sqrt{S_{1}^{2}+\cdots+S_{n}^{2}}} \sum_{j=1}^{n}\left(\mathbb{X}_{j}+\mathbb{Y}_{j}\right)$ has the unique limit determined by the law with Cauchy-Stieltjes transform (3.4). To do so, denote

$$
\mathbb{U}_{n}=\frac{1}{\sqrt{n}} \sum_{j=1}^{n} \mathbb{X}_{j}, \mathbb{V}_{n}=\frac{1}{\sqrt{n}} \sum_{j=1}^{n} \mathbb{Y}_{j}, \mathbb{S}_{n}=\mathbb{U}_{n}+\mathbb{V}_{n}
$$

Denote

$$
\mathbb{Z}_{j}^{(\varepsilon)}=\mathbb{X}_{j}^{\varepsilon} \mathbb{Y}_{j}^{1-\varepsilon}, \varepsilon=0,1
$$

Since the variables do not commute, we adopt a special convention for the product notation convention which relies on the order of the index set:

$$
\varphi\left(\prod_{s=1}^{p} \mathbb{Z}_{J(s)}^{\varepsilon(s)}\right):=\varphi\left(\mathbb{Z}_{J(1)}^{\varepsilon(1)} \mathbb{Z}_{J(2)}^{\varepsilon(2)} \ldots \mathbb{Z}_{J(p)}^{\varepsilon(p)}\right) .
$$

Lemma 4.3. In the above setting, if $\left(\mathbb{X}_{j}\right)$ satisfies Kargin's Condition $A$, then $\left\{\mathbb{X}_{1}, \mathbb{Y}_{1}, \mathbb{X}_{2}, \mathbb{Y}_{2}, \ldots\right\}$ satisfies Kargin's Condition $A$.

Proof. We first note the following.

Claim 1. Singleton properties (4.1), (4.2) for $\left\{\mathbb{X}_{j}\right\}$ are equivalent to the following: for $k \notin\left\{j_{1}, \ldots, j_{p}\right\}$ with $p=0,1,2, \ldots$, we have

$$
\begin{array}{r}
R_{p+1}\left(\mathbb{X}_{k}, \mathbb{X}_{j_{1}}, \ldots, \mathbb{X}_{j_{p}}\right)=R_{p+1}\left(\mathbb{X}_{j_{1}} \mathbb{X}_{k}, \mathbb{X}_{j_{2}}, \ldots, \mathbb{X}_{j_{p}}\right)=\ldots \\
\cdots=R_{p+1}\left(\mathbb{X}_{j_{1}}, \ldots, \mathbb{X}_{j_{p}}, \mathbb{X}_{k}\right)=0
\end{array}
$$

and

$$
r_{p+1}\left(\mathbb{X}_{k}, \mathbb{X}_{j_{1}}, \ldots, \mathbb{X}_{j_{p}}\right)=0
$$


Proof. Clearly, (4.7) implies (4.2) by (1.1) applied to $\varphi=\psi$. Conversely, suppose that $r_{p+1}\left(\mathbb{X}_{k}, \mathbb{X}_{j_{1}}, \ldots, \mathbb{X}_{j_{p}}\right) \neq 0$ for some $p \geq 0$, and take the smallest $p$. Since for $F=\left\{f_{1}, f_{2}, \ldots\right\} \subset\left\{j_{1}, \ldots, j_{p}\right\}$,

$$
\psi\left(\mathbb{X}_{k} \prod_{f \in F} \mathbb{X}_{f}\right)=0
$$

the only non-zero terms in (1.1) must come from cumulants that have $\mathbb{X}_{k}$ as their argument. Thus, with $\Pi_{F}$ denoting the appropriate products of moments,

$$
\begin{aligned}
0=\psi\left(\mathbb{X}_{k}, \mathbb{X}_{j_{1}} \ldots\right. & \left.\mathbb{X}_{j_{p}}\right)=\sum_{F} r_{|F|+1}\left(\mathbb{X}_{k}, \mathbb{X}_{f_{1}}, \mathbb{X}_{f_{2}}, \ldots\right) \Pi_{F} \\
& =r_{p+1}\left(\mathbb{X}_{k}, \mathbb{X}_{j_{1}}, \ldots, \mathbb{X}_{j_{p}}\right)+\text { lower order terms. }
\end{aligned}
$$

Since by assumption all lower order cumulants vanish, we see that $r_{p+1}\left(\mathbb{X}_{k}, \mathbb{X}_{j_{1}}, \ldots, \mathbb{X}_{j_{p}}\right)$ in fact must be zero.

Claim 2. Suppose $\left\{\mathbb{X}_{j}\right\}$ satisfies singleton properties (4.1) and (4.2). Then (4.3) is equivalent to the following: for $k \notin\left\{j_{1}, \ldots, j_{r}\right\}$ with $r=1,2, \ldots$, and every $0 \leq p \leq r$ we have

$$
R_{r+2}\left(\mathbb{X}_{k}, \mathbb{X}_{j_{1}}, \ldots, \mathbb{X}_{j_{p}}, \mathbb{X}_{k}, \mathbb{X}_{j_{p+1}} \ldots, \mathbb{X}_{j_{r}}\right)=0
$$

Proof. Suppose (4.6) and (4.9) hold. Then in (1.1), $\mathbb{X}_{k}$ must appear twice in the argument of $R$. Thus

$$
\varphi\left(\mathbb{X}_{k} \mathbb{X}_{j_{1}} \ldots \mathbb{X}_{j_{p}} \mathbb{X}_{k} \mathbb{X}_{j_{p+1}} \ldots \mathbb{X}_{j_{r}}\right)
$$

$=R_{2}\left(\mathbb{X}_{k}, \mathbb{X}_{k}\right) \psi\left(\mathbb{X}_{j_{1}} \ldots \mathbb{X}_{j_{p}}\right) \varphi\left(\mathbb{X}_{j_{p+1}} \ldots \mathbb{X}_{j_{r}}\right)+$ sum involving higher cumulants

$$
=\varphi\left(\mathbb{X}_{k}^{2}\right) \psi\left(\mathbb{X}_{j_{1}} \ldots \mathbb{X}_{j_{p}}\right) \varphi\left(\mathbb{X}_{j_{p+1}} \ldots \mathbb{X}_{j_{r}}\right)+0
$$

Conversely, suppose that $R_{r+2}\left(\mathbb{X}_{k}, \mathbb{X}_{j_{1}}, \ldots, \mathbb{X}_{j_{p}}, \mathbb{X}_{k}, \mathbb{X}_{j_{p+1}} \ldots, \mathbb{X}_{j_{r}}\right) \neq 0$, for some $r \geq 1$, and take the smallest such $r$. By (4.6), expansion (1.1) has no singleton appearances of $\mathbb{X}_{k}$. Thus

$$
\begin{gathered}
\varphi\left(\mathbb{X}_{k} \mathbb{X}_{j_{1}} \ldots \mathbb{X}_{j_{p}} \mathbb{X}_{k} \mathbb{X}_{j_{p+1}} \ldots \mathbb{X}_{j_{r}}\right)=R_{r+2}\left(\mathbb{X}_{k}, \mathbb{X}_{j_{1}}, \ldots, \mathbb{X}_{j_{p}}, \mathbb{X}_{k}, \mathbb{X}_{j_{p+1}} \ldots, \mathbb{X}_{j_{r}}\right) \\
+\sum_{\alpha=0}^{r-1} \sum_{\# F=\alpha} R_{\alpha+2}\left(\mathbb{X}_{k}, \mathbb{X}_{f_{1}}, \ldots, \mathbb{X}_{f_{a}}, \mathbb{X}_{k}, \mathbb{X}_{f_{a+1}}, \ldots\right) \\
=R_{r+2}\left(\mathbb{X}_{k}, \mathbb{X}_{j_{1}}, \ldots, \mathbb{X}_{j_{p}}, \mathbb{X}_{k}, \mathbb{X}_{j_{p+1}} \ldots, \mathbb{X}_{j_{r}}\right) \\
+R_{2}\left(\mathbb{X}_{k}, \mathbb{X}_{k}\right) \psi\left(\mathbb{X}_{j_{1}} \ldots \mathbb{X}_{j_{p}}\right) \varphi\left(\mathbb{X}_{j_{p+1}} \ldots \mathbb{X}_{j_{r}}\right) .
\end{gathered}
$$

Thus $R_{r+2}\left(\mathbb{X}_{k}, \mathbb{X}_{j_{1}}, \ldots, \mathbb{X}_{j_{p}}, \mathbb{X}_{k}, \mathbb{X}_{j_{p+1}} \ldots, \mathbb{X}_{j_{r}}\right)=0$ 
We will show that $\left\{\mathbb{Z}_{j}^{\varepsilon(j)}\right\}$ satisfies Kargin's Condition A for any choice of indices $(j, \varepsilon(j)) \in \mathbb{N} \times\{0,1\}$. Since the assumptions are symmetric with respect to $\left\{\mathbb{X}_{j}\right\}$ and $\left\{\mathbb{Y}_{j}\right\}$, it is enough to analyze the case when the distinguished element is $\mathbb{X}_{k}=\mathbb{Z}_{k}^{(1)}$.

Suppose $(1, k) \notin\left\{\left(\varepsilon(1), j_{1}\right),\left(\varepsilon(2), j_{2}\right), \ldots\left(\varepsilon(p), j_{p}\right)\right\}$. Then

$$
r_{p+1}\left(\mathbb{X}_{k}, \mathbb{Z}_{j_{1}}^{\varepsilon(1)}, \ldots, \mathbb{Z}_{j_{p}}^{\varepsilon(p)}\right)=0 \text {. }
$$

Indeed, this holds true by $\psi$-freeness of $\mathcal{A}_{\mathbb{X}}, \mathcal{A}_{\mathbb{Y}}$ if one of the $\varepsilon(i)=$ 0 . On the other hand, if all $\varepsilon(i)=1$, then this holds true by (4.6). Similarly, $(\varphi \mid \psi)$-freeness of $\mathcal{A}_{\mathbb{X}}, \mathcal{A}_{\mathbb{Y}}$ implies that

$$
R_{p+1}\left(\mathbb{X}_{k}, \mathbb{Z}_{j_{1}}^{\varepsilon(1)}, \ldots, \mathbb{Z}_{j_{p}}^{\varepsilon(p)}\right)=0
$$

either because some of the $\varepsilon(j)=0$, or by (4.6). Thus (4.1) and (4.2) hold for $\left\{\mathbb{Z}_{j}^{\varepsilon(j)}\right\}\left\{\mathbb{Z}_{j}^{\varepsilon(j)}\right\}$ by Claim 1 .

Similarly, if $r \geq 1$,

$$
R_{r+2}\left(\mathbb{X}_{k}, \mathbb{Z}_{j_{1}}^{\varepsilon(1)}, \ldots, \mathbb{Z}_{j_{p}}^{\varepsilon(1+p)}, \mathbb{X}_{k}, \mathbb{Z}_{j_{p+1}}^{\varepsilon(p+1)} \ldots, \mathbb{Z}_{j_{r}}^{\varepsilon(r)}\right)=0
$$

either because some of $\varepsilon(i)=0$ and $\mathcal{A}_{\mathbb{X}}, \mathcal{A}_{\mathbb{Y}}$ are $(\varphi \mid \psi)$-free, or by (4.9). Therefore (4.3) holds for $\left\{\mathbb{Z}_{j}^{\varepsilon(j)}\right\}$ by Claim (2).

Lemma 4.4. For fixed $j, k, m \geq 0$,

$$
\sup _{n}\left|\varphi\left(\mathbb{U}_{n}^{j} \mathbb{V}_{n}^{k}\left(\mathbb{U}_{n}+\mathbb{V}_{n}\right)^{m}\right)\right|<\infty
$$

Proof. Expanding the product, by Lemma 4.3 we see that

$$
\begin{aligned}
\varphi\left(\mathbb{U}_{n}^{j} \mathbb{V}_{n}^{k}\left(\mathbb{U}_{n}+\mathbb{V}_{n}\right)^{m}\right) & n^{-(j+k+m) / 2} \sum_{J:\{1, \ldots, j+k+m\} \rightarrow\{1, \ldots, n\}} \sum_{\varepsilon \in \mathcal{E}} \varphi\left(\prod_{s=1}^{j+k+m} \mathbb{Z}_{J(s)}^{\varepsilon(s)}\right) \\
= & n^{-(j+k+m) / 2} \sum_{J \in \mathcal{J}_{\geq 2}} \sum_{\varepsilon \in \mathcal{E}} \varphi\left(\prod_{s=1}^{j+k+m} \mathbb{Z}_{J(s)}^{\varepsilon(s)}\right),
\end{aligned}
$$

where

$$
\mathcal{J}_{\geq 2}=\left\{J: \# J^{-1}(s) \neq 1 \text { for all } 1 \leq s \leq n\right\}
$$

is the set of mappings $J:\{1, \ldots, j+k+m\} \rightarrow\{1, \ldots, n\}$ that take no singleton values, and

$$
\begin{aligned}
\mathcal{E}=\{ & \varepsilon \in 2^{\{1, \ldots, j+k+m\}}: \\
& \varepsilon(1)=\cdots=\varepsilon(j)=1, \quad \varepsilon(j+1)=\cdots=\varepsilon(j+k)=0\} .
\end{aligned}
$$


The cardinality of the first set can be bounded above by $\# \mathcal{J}_{\geq 2} \leq$ $n^{(j+k+m) / 2}$, and $\# \mathcal{E}=2^{m}$, so by (4.4),

$$
\left|\sum_{J \in \mathcal{J}_{\geq 2}} \sum_{\varepsilon \in \mathcal{E}} \varphi\left(\prod_{s=1}^{j+k+m} \mathbb{Z}_{J(s)}^{\varepsilon(s)}\right)\right| \leq C_{j+k+m} 2^{m} n^{(j+k+m) / 2} .
$$

Let

$$
\mathcal{J}_{2}=\left\{J: \# J^{-1}(s)=0,2 \text { for all } 1 \leq s \leq n\right\}
$$

be the subset of $\mathcal{J}_{\geq 2}$ that consists of all mappings $J:\{1, \ldots, j+k+$ $m\} \rightarrow\{1, \ldots, n\}$ that are two-to-one valued. (Clearly $\mathcal{J}_{2}=\emptyset$ when $j+k+m$ is odd.)

Lemma 4.5. For $j, k, m \geq 0$,

$$
\limsup _{n \rightarrow \infty}\left|\varphi\left(\mathbb{U}_{n}^{j} \mathbb{V}_{n}^{k}\left(\mathbb{U}_{n}+\mathbb{V}_{n}\right)^{m}\right)-n^{-(j+k+m) / 2} \sum_{J \in \mathcal{J}_{2}} \sum_{\varepsilon \in \mathcal{E}} \varphi\left(\prod_{s=1}^{j+k+m} \mathbb{Z}_{J(s)}^{\varepsilon(s)}\right)\right|=0,
$$

Proof. If there is a value $s \in\{1 \ldots n\}$ that is taken by $J$ at three or more different points, then there are at most $j+k+m-1$ points on which $J$ is two-to-one. Therefore,

$$
\#\left(\mathcal{J}_{\geq 2} \backslash \mathcal{J}_{2}\right) \leq\left(\begin{array}{c}
j+k+m \\
3
\end{array}\right) n^{(j+k+m-1) / 2},
$$

and by (4.4), the result follows from (4.12),

$$
\sum_{J \in \mathcal{J}_{\geq 2} \backslash \mathcal{J}_{2}} \sum_{\varepsilon}\left|\varphi\left(\prod_{s=1}^{j+k+m} \mathbb{Z}_{J(s)}^{\varepsilon(s)}\right)\right| \leq\left(\begin{array}{c}
j+k+m \\
3
\end{array}\right) C_{j+k+m} 2^{m} n^{(j+k+m-1) / 2} .
$$

We remark that since $\mathcal{J}_{2}=\emptyset$ for odd $j+k+m$, Lemma 4.5 implies that

$$
\limsup _{n \rightarrow \infty}\left|\varphi\left(\left(\mathbb{U}_{n}+\mathbb{V}_{n}\right)^{m}\right)\right|=0 \text { for odd } m .
$$

The next lemma is the main tool in identifying the limit via Theorem 2.1 .

Lemma 4.6. For $m \geq 1$,

$$
\limsup _{n \rightarrow \infty}\left|\varphi\left(\left(\mathbb{U}_{n}-\mathbb{V}_{n}\right)^{2} \mathbb{S}_{n}^{m}\right)-2 \varphi\left(\mathbb{S}_{n}^{m}\right) \sum_{j=1}^{n} S_{j}^{2} / n\right|=0
$$


Proof. Since $(x-y)^{2}=x(x-y)+y(y-x)$, and the joint moments of $\left(\mathbb{U}_{n}, \mathbb{V}_{n}\right)$ are symmetric in $\mathbb{U}_{n}, \mathbb{V}_{n}$, it is enough to show that

$$
\limsup _{n \rightarrow \infty}\left|\varphi\left(\mathbb{U}_{n}\left(\mathbb{U}_{n}-\mathbb{V}_{n}\right) \mathbb{S}_{n}^{m}\right)-\varphi\left(\mathbb{S}_{n}^{m}\right) \sum_{j=1}^{n} S_{j}^{2} / n\right|=0
$$

By Lemma 4.5, once we expand the sums in $\varphi\left(\mathbb{U}_{n}^{2} \mathbb{S}_{n}^{m}-\mathbb{V}_{n} \mathbb{S}_{n}^{m}-\mathbb{S}_{n}^{m} \sum_{j=1}^{n} S_{j}^{2} / n\right)$, the only contributing terms come from the sum over the two-to-one functions $J:\{1 \ldots m+2\} \rightarrow\{1 \ldots n\}$. Therefore, it is enough to show that before taking the limit, we have the following identity:

$$
\begin{array}{r}
\frac{1}{n} \sum_{J \in J_{2}} \sum_{\varepsilon}\left(\varphi\left(\mathbb{X}_{J(1)} \mathbb{X}_{J(2)} \prod_{s=3}^{m+2} \mathbb{Z}_{J(s)}^{\varepsilon(s)}\right)-\varphi\left(\mathbb{X}_{J(1)} \mathbb{Y}_{J(2)} \prod_{s=3}^{m+2} \mathbb{Z}_{J(s)}^{\varepsilon(s)}\right)\right. \\
\left.-S_{J(1)}^{2} \delta_{J(1)=J(2)} \varphi\left(\prod_{s=3}^{m+2} \mathbb{Z}_{J(s)}^{\varepsilon(s)}\right)\right)=0 .
\end{array}
$$

Let $\mathcal{J}_{*} \subset \mathcal{J}_{2}$ denote the set of two-to-one functions with $J(1)=J(2)$. Expanding the products we see that for $J \in \mathcal{J}_{*}$ each term in (4.17) can be written as

$$
\varphi\left(\mathbb{X}_{J(1)} \mathbb{X}_{J(2)} \prod_{s=3}^{m+2} \mathbb{Z}_{J(s)}^{\varepsilon(s)}\right)-\varphi\left(\mathbb{X}_{J(1)} \mathbb{Y}_{J(2)} \prod_{s=3}^{m+2} \mathbb{Z}_{J(s)}^{\varepsilon(s)}\right)-S_{J(1)}^{2} \varphi\left(\prod_{s=3}^{m+2} \mathbb{Z}_{J(s)}^{\varepsilon(s)}\right)
$$

Since $\mathbb{Y}_{J(2)}$ is a singleton, by Lemma 4.3, $\varphi\left(\mathbb{X}_{J(1)} \mathbb{Y}_{J(2)} \prod_{s=3}^{m+2} \mathbb{Z}_{J(s+2)}^{\varepsilon(s)}\right)=$ 0 . The same lemma gives

$$
\varphi\left(\mathbb{X}_{J(1)} \mathbb{X}_{J(2)} \prod_{s=3}^{m+2} \mathbb{Z}_{J(s)}^{\varepsilon(s)}\right)=\varphi\left(\mathbb{X}_{J(1)}^{2} \prod_{s=3}^{m+2} \mathbb{Z}_{J(s)}^{\varepsilon(s)}\right)=S_{J(1)}^{2} \varphi\left(\prod_{s=3}^{m+2} \mathbb{Z}_{J(s)}^{\varepsilon(s)}\right)
$$

Thus

$$
\begin{array}{r}
\sum_{J \in \mathcal{J}_{*}} \sum_{\varepsilon}\left(\varphi\left(\mathbb{X}_{J(1)} \mathbb{X}_{J(2)} \prod_{s=3}^{m+2} \mathbb{Z}_{J(s)}^{\varepsilon(s)}\right)-\varphi\left(\mathbb{X}_{J(1)} \mathbb{Y}_{J(2)} \prod_{s=3}^{m+2} \mathbb{Z}_{J(s)}^{\varepsilon(s)}\right)\right) \\
=\varphi\left(\mathbb{S}_{n}^{m}\right) \sum_{j=1}^{n} S_{j}^{2}
\end{array}
$$


To end the proof, we need to show that the sum over $J \in \mathcal{J}_{2} \backslash \mathcal{J}_{*}$ is zero. In fact, we observe that for each $J \in \mathcal{J}_{2} \backslash \mathcal{J}_{*}$,

$$
\sum_{\varepsilon}\left(\varphi\left(\mathbb{X}_{J(1)} \mathbb{X}_{J(2)} \prod_{s=3}^{m+2} \mathbb{Z}_{J(s)}^{\varepsilon(s)}\right)-\varphi\left(\mathbb{X}_{J(1)} \mathbb{Y}_{J(2)} \prod_{s=3}^{m+2} \mathbb{Z}_{J(s)}^{\varepsilon(s)}\right)\right)=0 .
$$

To see this, denote by $r>2$ the unique index with $J(1)=J(r)$. Given $\varepsilon \in 2^{\{3, \ldots, m+2\}}$, let

$$
\varepsilon^{\prime}(s)= \begin{cases}1-\varepsilon(s) & \text { if } s<r \\ \varepsilon(s) & \text { if } s \geq r\end{cases}
$$

Clearly, the mapping $\varepsilon \mapsto \varepsilon^{\prime}$ is a bijection of $\mathcal{E}$. Therefore, (4.19) follows from

$$
\sum_{\varepsilon}\left(\varphi\left(\mathbb{X}_{J(1)} \mathbb{X}_{J(2)} \prod_{s=3}^{m+2} \mathbb{Z}_{J(s)}^{\varepsilon(s)}\right)=\sum_{\varepsilon} \varphi\left(\mathbb{X}_{J(1)} \mathbb{Y}_{J(2)} \prod_{s=3}^{m+2} \mathbb{Z}_{J(s)}^{\varepsilon^{\prime}(s)}\right)\right)
$$

The latter holds true because by Lemma 4.3, for a fixed $\varepsilon$, the left hand side of (4.20) is

$$
\varphi\left(\mathbb{X}_{J(1)}^{2}\right) \psi\left(\mathbb{X}_{J(2)} \prod_{s=3}^{r-1} \mathbb{Z}_{J(s)}^{\varepsilon(s)}\right) \varphi\left(\prod_{s=r+1}^{m+2} \mathbb{Z}_{J(s)}^{\varepsilon(s)}\right)
$$

while the right hand side of (4.20) is

$$
\varphi\left(\mathbb{X}_{J(1)}^{2}\right) \psi\left(\mathbb{Y}_{J(2)} \prod_{s=3}^{r-1} \mathbb{Z}_{J(s)}^{\varepsilon^{\prime}(s)}\right) \varphi\left(\prod_{s=r+1}^{m+2} \mathbb{Z}_{J(s)}^{\varepsilon(s)}\right)
$$

The two expressions are equal, because the joint (mixed) $\psi$-moments of $\mathbb{X}_{1}, \mathbb{X}_{2}, \ldots, \mathbb{Y}_{1}, \mathbb{Y}_{2}, \ldots$ by construction do not change when we swap the roles of the sequences $\left\{\mathbb{X}_{j}\right\}$ and $\left\{\mathbb{Y}_{j}\right\}$. Of course, such a transformation converts $\psi\left(\mathbb{X}_{J(2)} \prod_{s=3}^{r-1} \mathbb{Z}_{J(s)}^{\varepsilon(s)}\right)$ into $\psi\left(\mathbb{Y}_{J(2)} \prod_{s=3}^{r-1} \mathbb{Z}_{J(s)}^{\varepsilon^{\prime}(s)}\right)$.

Proof of Theorem 4.1. Since convergence of moments is a metric convergence, we use the standard lemma: to show convergence it suffices to show that every subsequence has a subsequence that converges to the same limit.

The joint $\psi$-moments of $\mathbb{U}_{n}, \mathbb{V}_{n}, \mathbb{S}_{n}$ converge, as the $\psi$-moments of $\mathbb{U}_{n}$ converge by assumption and (4.5), and $\mathbb{U}_{n}, \mathbb{V}_{n}$ are $\psi$-free so their joint $\psi$-moments are uniquely determined from the moments of $\mathbb{U}_{n}$ alone.

By Lemma 4.4, from any subsequence $\mathbb{U}_{n_{k}}$ by diagonal method we can extract a further sub-subsequence such that the joint $\varphi$-moments of 
$\mathbb{U}_{n}, \mathbb{V}_{n}$, and $\mathbb{S}_{n}$ converge along that sub-subsequence. Taken together, the limits of these $\psi$-moments and $\varphi$-moments define a pair of states on $\mathbb{C}\langle\mathbb{U}, \mathbb{V}\rangle$, which we will denote again by $\psi$ and $\varphi$. Since $\mathbb{U}_{n}, \mathbb{V}_{n}$ are $\psi$-free and $(\varphi \mid \psi)$-free under the limit state $\mathbb{U}, \mathbb{V}$ are also $\psi$-free and $(\varphi \mid \psi)$-free. From Lemma 4.6, we see that the pair

$$
\mathbb{X}:=\mathbb{U} / S, \mathbb{Y}:=\mathbb{V} / S
$$

satisfies the assumptions of Theorem 2.1 with $a=b=0$. By Proposition 3.1 , this determines the $\varphi$-law of $\mathbb{U}$ uniquely. Therefore, the original sequence $\left\{\mathbb{U}_{n}\right\}$ converges in $\varphi$-moments to $\mathbb{U}$, and the $\varphi$-law of $\frac{1}{\sqrt{S_{1}^{2}+\cdots+S_{n}^{2}}} \sum_{j=1}^{n} \mathbb{X}_{j}=\sqrt{\frac{n}{S_{1}^{2}+\cdots+S_{n}^{2}}} \mathbb{U}_{n}$ converges in $\varphi$-moments to $\mathbb{U} / S$. Since the $\psi$-law of $\mathbb{U} / S$ is the dilations by $s / S$ of measure $\nu$, we get formula (3.6).

Proof of Corollary 4.2. By 7], or by repeating the proof of Theorem 4.1 in the special case when $\varphi=\psi$ with [1] used instead of Theorem 2.1, we know that the $\psi$-moments of $\frac{1}{\sqrt{S_{1}^{2}+\cdots+S_{n}^{2}}} \sum_{j=1}^{n} \mathbb{X}_{j}$ converge to the semicircle law of variance $\sigma^{2}=s^{2} / S^{2}$.

Since the semicircle law has compact support, we can use Theorem 4.1, the limiting distribution is then given by (3.7).

Acknowledgements. The second named author thanks M. Peligrad for bringing ref. [7] to his attention.

\section{REFERENCES}

[1] Bożejko, M., And Bryc, W. On a class of free Lévy laws related to a regression problem. J. Funct. Anal. 236 (2006), 59-77. arxiv.org/abs/math.OA/0410601.

[2] Bożejko, M., Leinert, M., And Speicher, R. Convolution and limit theorems for conditionally free random variables. Pacific J. Math. 175, 2 (1996), $357-388$.

[3] Bożejko, M., AND Speicher, R. $\psi$-independent and symmetrized white noises. In Quantum probability \& related topics, QP-PQ, VI. World Sci. Publishing, River Edge, NJ, 1991, pp. 219-236.

[4] Bożejko, M., And Wysoczański, J. New examples of convolutions and noncommutative central limit theorems. In Quantum probability (Gdańsk, 1997), vol. 43 of Banach Center Publ. Polish Acad. Sci., Warsaw, 1998, pp. 95-103.

[5] Bożejko, M., And Wysoczański, J. Remarks on $t$-transformations of measures and convolutions. Ann. Inst. H. Poincaré Probab. Statist. 3\%, 6 (2001), $737-761$.

[6] Bryc, W., AND WesoŁowski, J. Bi-Poisson process. Infin. Dimens. Anal. Quantum Probab. Relat. Top. 10 (2007), 277-291. arxiv.org/abs/math.PR/0404241. 
[7] Kargin, V. A proof of a non-commutative central limit theorem by the Lindeberg method. Electron. Comm. Probab. 12 (2007), 36-50 (electronic).

[8] Krystek, A., And Yoshida, H. The combinatorics of the $r$-free convolution. Infin. Dimens. Anal. Quantum Probab. Relat. Top. 6, 4 (2003), 619-627.

[9] Krystek, A., And Yoshida, H. Generalized $t$-transformations of probability measures and deformed convolutions. Probab. Math. Statist. 24, 1, Acta Univ. Wratislav. No. 2646 (2004), 97-119.

[10] Lehner, F. Cumulants in noncommutative probability theory. I. Noncommutative exchangeability systems. Math. Z. 248, 1 (2004), 67-100.

[11] NicA, A., AND Speicher, R. Lectures on the combinatorics of free probability, vol. 335 of London Mathematical Society Lecture Note Series. Cambridge University Press, Cambridge, 2006.

[12] Speicher, R. Multiplicative functions on the lattice of noncrossing partitions and free convolution. Math. Ann. 298, 4 (1994), 611-628.

[13] Speicher, R. Free probability theory and non-crossing partitions. Sém. Lothar. Combin. 39 (1997), Art. B39c, 38 pp. (electronic).

[14] Voiculescu, D. Addition of certain noncommuting random variables. $J$. Funct. Anal. 66, 3 (1986), 323-346.

Instytut Matematyczny, Uniwersytet Wroceawski, Pl. Grunwaldzki 2/4 50-384 Wroceaw, Poland.

E-mail address: bozejko@math.uni.wroc.pl

Department of Mathematics, University of Cincinnati, PO Box 210025, Cincinnati, OH 45221-0025, USA

E-mail address: Wlodzimierz.Bryc@UC.edu 